\title{
Metal-cluster ionization energy: A profile-insensitive exact expression for the size effect
}

\author{
Michael Seidl and John P. Perdew \\ Department of Physics and Quantum Theory Group, Tulane University, New Orleans, Louisiana 70118 \\ Marta Brajczewska and Carlos Fiolhais \\ Departamento de Física da Universidade de Coimbra, P-3000 Coimbra, Portugal
}

(Received 18 November 1996)

\begin{abstract}
The ionization energy of a large spherical metal cluster of radius $R$ is $I(R)=W+\left(\frac{1}{2}+c\right) / R$, where $W$ is the bulk work function and $c \approx-0.1$ is a material-dependent quantum correction to the electrostatic size effect. We present "Koopmans" and "displaced-profile change-in-self-consistent-field" expressions for $W$ and $c$ within the ordinary and stabilized-jellium models. These expressions are shown to be exact and equivalent when the exact density profile of a large neutral cluster is employed; these equivalences generalize the BuddVannimenus theorem. With an approximate profile obtained from a restricted variational calculation, the "displaced-profile", expressions are the more accurate ones. This profile insensitivity is important, because it is not practical to extract $c$ from solutions of the Kohn-Sham equations for small metal clusters. [S0163-1829(97)01219-8]
\end{abstract}

The ionization energy $I(R)$ of a spherical metal cluster with radius $R$ is the work needed to remove an electron from the neutral cluster. By definition, $R=r_{s} N^{1 / 3}$, where $r_{s}$ is the Seitz radius or bulk density parameter of the corresponding bulk metal, and $N$ is the number of valence electrons in the neutral cluster. For large radii $R$, the ionization energy can be expanded as ${ }^{1-3}$

$$
I(R)=W+\left(\frac{1}{2}+c\right) \frac{1}{R}+O\left(R^{-2}\right) .
$$

We use atomic units $\left(e^{2}=\hbar=m=1\right) . W$ is the bulk work function of the planar metal surface, corresponding to the limit $R \rightarrow \infty$. $c \approx-0.1$ is a material-dependent quantum correction to the classical value $1 / 2$ (Ref. 4 ) of the $R^{-1}$ coefficient in the expansion (1). The electron affinity $A(R)$ of a large cluster is given ${ }^{1}$ by the right-hand side of Eq. (1) with the substitution $1 / 2 \rightarrow-1 / 2$.

In the jellium model ${ }^{5-7}$ there are two expressions for $W$ (Refs. 5 and 8-10) and two for $c$ (Refs. 11 and 12, and Ref. 3 ) in terms of the density profile of the neutral cluster. The Mahan-Schaich ${ }^{8}$ or "displaced-profile change-in-selfconsistent-field" (Refs. 9 and 10) expression for $W$ is equivalent via the Budd-Vannimenus theorem ${ }^{13}$ to the exact "Koopmans", expression of Lang and Kohn," but is less sensitive $^{9,10}$ to errors in the approximate density profile for the neutral planar surface. Here we similarly show for the size-effect coefficient $c$ that the Seidl-Brack or "displacedprofile change-in-self-consistent-field', expression, derived as an approximation in Ref. 3, is exact, like the "Koopmans" expression of Pogosov and co-workers, ${ }^{11,12}$ but is less sensitive to errors in the approximate density profile of the neutral finite cluster.

In the jellium model, ${ }^{5-7}$ the total energy as a functional $^{14,15}$ of the electron density $n(\mathbf{r})$ is

$$
\begin{aligned}
E_{R}[n]= & T_{s}[n]+E_{\mathrm{xc}}[n]+\frac{1}{2} \int d^{3} r \\
& \times \int d^{3} r^{\prime} \frac{\left[n(\mathbf{r})-n_{+R}(\mathbf{r})\right]\left[n\left(\mathbf{r}^{\prime}\right)-n_{+R}\left(\mathbf{r}^{\prime}\right)\right]}{\left|\mathbf{r}-\mathbf{r}^{\prime}\right|},
\end{aligned}
$$

where $n_{+R}(r)=\bar{n} \Theta(R-r)$ is the uniform positive background density, and

$$
\bar{n}=\frac{3}{4 \pi r_{s}^{3}}
$$

$T_{s}$ and $E_{\mathrm{xc}}$ are the noninteracting kinetic and the exchange-correlation ${ }^{15}$ energies, respectively.

We begin with a review of the derivation of the "Koopmans" expressions. We consider continuum densityfunctional approximations such as the gradient expansion ${ }^{14}$ for $T_{s}[n]$ or the local-density approximation ${ }^{15}$ for $E_{\mathrm{xc}}[n]$, for which we can write ${ }^{1,2,16}$

$$
I(R)=-\mu(R)+\frac{1}{2 R}+O\left(R^{-2}\right)
$$

where

$$
\mu(R)=\left.\frac{\delta E_{R}[n]}{\delta n(\mathbf{r})}\right|_{n=n_{R}}=\phi_{R}(\mathbf{r})+\left.\frac{\delta E_{k \mathrm{xc}}[n]}{\delta n(\mathbf{r})}\right|_{n=n_{R}}
$$

is the chemical potential of a neutral cluster with radius $R$. Equation (4) is the Euler equation of density functional theory. ${ }^{14}$ In Eq. (4), $n_{R}(\mathbf{r})$ is the exact ground-state density,

$$
\phi_{R}(\mathbf{r})=\int d^{3} r^{\prime} \frac{\left[n\left(\mathbf{r}^{\prime}\right)-n_{+R}\left(\mathbf{r}^{\prime}\right)\right]}{\left|\mathbf{r}-\mathbf{r}^{\prime}\right|}
$$

is the electrostatic potential energy of an electron, and $E_{k \mathrm{xc}}$ $=T_{s}+E_{\mathrm{xc}}$. 
Because of the Hohenberg-Kohn theorem, ${ }^{14} \mu(R)$ is a constant, independent of $\mathbf{r}$. Let us evaluate the right-hand side of Eq. (4) at a point $\mathbf{r}$ deep inside the cluster where $n_{R}(\mathbf{r})=\bar{n}$, and

$$
\left.\frac{\delta E_{k \mathrm{xc}}[n]}{\delta n(\mathbf{r})}\right|_{n=n_{R}}=\frac{d}{d \bar{n}}\left[\bar{n} e_{k \mathrm{xc}}(\bar{n})\right]=\mu_{k \mathrm{xc}}(\bar{n}) .
$$

$e_{k \mathrm{xc}}(\bar{n})$ is the sum of the noninteracting kinetic and exchange-correlation energies per electron in a uniform gas of density $\bar{n}$. We expand ${ }^{2} \phi_{R}(\mathbf{r})$ about the electrostatic potential $\phi(x)$ of the planar surface,

$$
\phi_{R}(x)=\phi(x)+\frac{h(x)}{R}+O\left(R^{-2}\right),
$$

where $x=r-R$ gives the distance from the edge of the spherical positive background. From Eq. (5), $\phi(\infty)=h(\infty)$ $=0$ and, for $x$ deep inside the cluster, $\phi(x)$ and $h(x)$ can be replaced by the constants $\phi(-\infty)$ and $h(-\infty)$, respectively. Combining Eq. (1) with Eqs. (3), (4), (6), and (7), we find the familiar "Koopmans" expression

$$
\begin{aligned}
W & =\Delta \phi-\mu_{k \mathrm{xc}}(\bar{n}) \\
& =4 \pi \int_{-\infty}^{\infty} d x x[n(x)-\bar{n} \Theta(-x)]-\mu_{k \mathrm{xc}}(\bar{n})
\end{aligned}
$$

for the bulk work function, where $\Delta \phi=-\phi(-\infty)$ is the electrostatic surface dipole barrier. We also find

$$
c=-h(-\infty)=4 \pi \int_{-\infty}^{\infty} d x\left\{x^{2}[n(x)-\bar{n} \Theta(-x)]+x f(x)\right\},
$$

where $f(x)$ is the $R^{-1}$ coefficient in the expansion ${ }^{2}$

$$
n_{R}(x)=n(x)+\frac{f(x)}{R}+O\left(R^{-2}\right)
$$

of the density profile $n_{R}(x)$ of the neutral cluster about the profile $n(x)$ of the planar metal surface. $n(x)$ and $f(x)$ are to be found by extrapolating $n_{R}(x)$ and $R\left[n_{R}(x)-n(x)\right]$, respectively, to $R=\infty$. To derive the right-hand side of Eq. (9), we used Eq. (A9) in Ref. 2 for the function $h(x)$, and integrated by parts. Equation (9) was earlier derived in Refs. 11 and 12.

Expressions (8) and (9) are simply understood from the exact result $\mu=\epsilon_{F}$, where $\epsilon_{F}$ is the highest occupied Kohn-Sham ${ }^{15}$ orbital energy. We call these expressions "Koopmans-like," because of the resemblance to Koopmans' theorem. Note that their derivation is based on the constancy of the right-hand side of Eq. (4) over all space. However, this constancy can fail if Eq. (4) is evaluated with an approximate density profile instead of the exact solution of the Euler equation.

Alternative expressions for $W$ and $c$ can be found from the liquid drop model ${ }^{2,3,17-19}$ for the total energy of the cluster,

$$
E=\left(4 \pi R^{2} \Sigma\right)^{2} / 2 R+\alpha 4 \pi R^{3} / 3+\sigma 4 \pi R^{2}+\gamma 2 \pi R+\cdots,
$$

where $\alpha, \sigma$, and $\gamma$ are, respectively, the volume, surface, and curvature energies. The chemical potential is $\mu=\partial E / \partial N$. Since all the excess charge in a metal resides on the surface, the change in the electron number is $d N=-4 \pi R^{2} d \Sigma$, where $\Sigma$ is the surface charge density. For the same reason, only $\sigma$ and $\gamma$ in Eq. (11) depend on $\Sigma$, while $\alpha$ is charge independent. Comparing with Eqs. (1) and (3), we find

$$
\begin{aligned}
& W=\left.\frac{d \sigma}{d \Sigma}\right|_{\Sigma=0}, \\
& c=\left.\frac{1}{2} \frac{d \gamma}{d \Sigma}\right|_{\Sigma=0} .
\end{aligned}
$$

We call these equations "change-in-self-consistent-field" expressions, since they are found directly from the change in the total energy upon ionization. Equation (12) was derived in Ref. 9, and Eq. (13) in Ref. 2. In Ref. 3, where $z=$ $-4 \pi R^{2} \Sigma$ is used as the charge variable, the charge dependence of the coefficients in expansion (11) is shifted to terms of higher order in $R^{-1}$. This expansion of Ref. 3 is easily rearranged by substituting $z \rightarrow-4 \pi R^{2} \Sigma$, and re-sorting the terms with respect to different powers of $R^{-1}$.

To evaluate Eqs. (12) and (13), we use a "displacedprofile" model ${ }^{3}$ for the electron-density profile of the charged cluster,

$$
n_{R, \Sigma}^{\mathrm{DP}}(x)=n_{R}\left(x-\delta_{R, \Sigma}\right),
$$

where $n_{R}(x)$ is the profile of the neutral cluster, and the displacement $\delta_{R, \Sigma}$ is chosen to satisfy

$$
4 \pi \int_{-R}^{\infty} d x(R+x)^{2}\left[n_{R}\left(x-\delta_{R, \Sigma}\right)-\bar{n} \Theta(-x)\right]=-4 \pi R^{2} \Sigma .
$$

Then Refs. 8 and 9 show that

$$
W=\Delta \phi^{>}-e_{k \mathrm{xc}}(\bar{n})=4 \pi \int_{0}^{\infty} d x x n(x)-e_{k \mathrm{xc}}(\bar{n}),
$$

where $\Delta \phi^{>}=-\phi(0)$ is the outer part of the surface dipole barrier $\Delta \phi$. Similarly, Ref. 3 shows that

$$
c=-\frac{2}{3} a_{s} r_{s}+4 \pi \int_{0}^{\infty} d x\left[x^{2} n(x)+x f(x)\right],
$$

where $a_{s}=4 \pi r_{s}^{2} \sigma(\Sigma=0)$.

To see that Eqs. (16) and (17) are exact, we only have to appeal to the variational principle ${ }^{14}$

$$
\begin{aligned}
E_{R}\left[n_{R, \Sigma}^{\mathrm{DP}}\right]= & E_{R}\left[n_{R, \Sigma}\right]+\frac{1}{2} \int d^{3} r \\
& \times\left.\int d^{3} r^{\prime} \frac{\delta^{2} E_{R}[n]}{\delta n(\mathbf{r}) \delta n\left(\mathbf{r}^{\prime}\right)}\right|_{n=n_{R, \Sigma}} \\
& \times \delta n(\mathbf{r}) \delta n\left(\mathbf{r}^{\prime}\right)+\cdots,
\end{aligned}
$$

where $\delta n(\mathbf{r})=n_{R, \Sigma}^{\mathrm{DP}}(x)-n_{R, \Sigma}(x)$ is of order $\Sigma$, since both $n_{R, \Sigma}^{\mathrm{DP}}(x)-n_{R}(x)$ and $n_{R, \Sigma}(x)-n_{R}(x)$ are of order $\Sigma$. Because the second term in Eq. (18) is of order $\Sigma^{2}$, it does not 
TABLE I. Comparison of the whole surface dipole barrier $\Delta \phi$ $=-\phi(-\infty)$ and its outer part $\Delta \phi^{>}=-\phi(0)$ and inner part $\Delta \phi^{<}=\phi(0)-\phi(-\infty)$ for the planar jellium surface, evaluated with exact Kohn-Sham (Ref. 5) and Seidl-Brack restricted variational (Ref. 3) density profiles ( $r_{s}$ in bohr, $\phi$ in $\mathrm{eV}$. 1 hartree $=27.21 \mathrm{eV})$. Since Refs. 5 and 3 employ different kineticenergy functionals and different parametrizations for $e_{c}(\bar{n})$, comparisons between them should not be made at too fine a level.

\begin{tabular}{ccccccc}
\hline \hline$r_{s}$ & $\Delta \phi_{\mathrm{KS}}$ & $\Delta \phi_{\mathrm{var}}$ & $\Delta \phi_{\mathrm{KS}}^{>}$ & $\Delta \phi_{\mathrm{var}}^{>}$ & $\Delta \phi_{\mathrm{KS}}^{<}$ & $\Delta \phi_{\mathrm{var}}^{<}$ \\
\hline 2 & 6.80 & 6.08 & 3.95 & 3.41 & 2.85 & 2.67 \\
3 & 2.32 & 2.11 & 1.58 & 1.24 & 0.74 & 0.87 \\
4 & 0.91 & 1.04 & 0.81 & 0.63 & 0.10 & 0.41 \\
5 & 0.35 & 0.63 & 0.48 & 0.39 & -0.13 & 0.24 \\
6 & 0.04 & 0.37 & 0.30 & 0.27 & -0.26 & 0.10 \\
\hline \hline
\end{tabular}

contribute to derivatives like those in Eqs. (12) and (13), i.e., use of the displaced profile introduces no error into $W$ or $c$.

For the $n(x)$ and $f(x)$, that solve the Euler equation (4), Eqs. (8) and (16) yield the same exact work function, while Eqs. (9) and (17) should yield the same exact size-effect coefficient $c$. Writing $\Delta \phi=\Delta \phi^{<}+\Delta \phi^{>}$, where $\Delta \phi^{<}$ $=\phi(0)-\phi(-\infty)$, and equating Eqs. (8) and (16), yields the Budd-Vannimenus ${ }^{13,8}$ theorem

$$
\Delta \phi^{<}=4 \pi \int_{-\infty}^{0} d x x[n(x)-\bar{n}]=\bar{n} \frac{d}{d \bar{n}} e_{k \mathrm{xc}}(\vec{n}) .
$$

Equating Eqs. (9) and (17) yields an expression for the surface energy,

$$
a_{s}=-\frac{6 \pi}{r_{s}} \int_{-\infty}^{0} d x\left\{x^{2}[n(x)-\bar{n} \Theta(-x)]+x f(x)\right\} .
$$

Note that this expression does not contain the bulk energy $e_{k \mathrm{xc}}(\bar{n})$, but presents the surface energy purely in terms of the profiles $n(x)$ and $f(x)$.

For approximate $n(x)$ and $f(x)$ obtained from a restricted variational calculation, the "displaced-profile change-in-selfconsistent-field' expressions (16) and (17) are expected to be the more accurate and less profile-sensitive expressions, although they are not extremal. ${ }^{9,10}$ Table I illustrates these observations via a comparison of results for the surface dipole barriers $\Delta \phi=-\phi(-\infty)$ and $\Delta \phi^{>}=-\phi(0)$ calculated for the planar surface of jellium with two different density profiles: the Lang-Kohn ${ }^{5}$ exact solution of the Kohn-Sham equations, and the Seidl-Brack ${ }^{3}$ restricted variational solution. The exact and restricted variational results for $\Delta \phi$ [which appears in Eq. (8)] are rather different, while those for $\Delta \phi^{>}$[which appears in Eq. (16)] are much more nearly equal.

To explain what we mean by "profile insensitivity," we suppose that the trial density profile for the planar surface is $n(x)=\bar{n} g\left(\gamma k_{s} x\right)$, where $g(y)$ is a shape function, $\gamma$ is a variational scaling parameter, and $k_{s}=(4 / \pi)^{1 / 2}\left(3 \pi^{2} \bar{n}\right)^{1 / 6}$ is the inverse of the Thomas-Fermi screening length. $W$ of Eq. (16) is not insensitive to $\gamma$, and in fact $\Delta \phi^{>} \propto \gamma^{-2}$. But, once $\gamma$ has been chosen to minimize the energy, $W$ of Eq. (16) is insensitive to the shape function $g(y)$ within the class of
TABLE II. Size-effect coefficient $c$ of Eq. (1) for jellium spheres, evaluated in various ways from the restricted variational calculation of Seidl and Brack (Ref. 3). The $I(R)$ values were found by extrapolation to $R=\infty$ of $R[I(R)-W]-\frac{1}{2}$, while the $\phi_{R}(-R)$ values were found by extrapolation of $-R\left[\phi_{R}(-R)-\phi(-\infty)\right]$. The profile-insensitive results from $I(R)$ or from Eq. (17) are the more accurate ones. The profile-sensitive results from $\phi_{R}(-R)$ or from Eq. (9) were not reported in Ref. 3. The energy functional includes the fourth-order gradient expansion for $T_{s}[n]$ and the local-density approximation for $E_{\mathrm{xc}}[n]$. The last column shows the results of an exact solution (Ref. 2) of the Euler equation for this functional.

\begin{tabular}{cccccc}
\hline \hline$r_{s}$ & $I(R)$ & Eq. (17) & $\phi_{R}(-R)$ & Eq. (9) & Euler \\
\hline 2 & -0.078 & -0.072 & -0.067 & -0.080 & -0.072 \\
3 & -0.085 & -0.082 & -0.049 & -0.057 & \\
4 & -0.084 & -0.083 & -0.034 & -0.035 & -0.083 \\
5 & -0.083 & -0.084 & -0.019 & -0.023 & \\
6 & -0.083 & -0.082 & -0.013 & -0.016 & -0.075 \\
\hline \hline
\end{tabular}

physically reasonable shapes. For example, very similar work functions are found for the three different approximate shape functions of Refs. 3 and 10, and for the exact KohnSham shape function of Refs. 5 and 8.

The profile sensitivity of the "Koopmans" expressions (8) and (9) can be traced in part to their dependence upon the density profiles inside the jellium edge $(x<0)$, where the profile $n(x)$ that solves the Euler equation must oscillate above $\bar{n}$ for low bulk densities $\bar{n}$. To see why, look at Eq. (19) for the inner part $\Delta \phi^{<}$of the surface dipole barrier. The integral for $\Delta \phi^{<}$in Eq. (19) cannot be negative unless $n(x)>\bar{n}$ for some negative $x$. Since $e_{k \times c}$ minimizes at $r_{s}$ $=4.2, \Delta \phi^{<}$must be negative for $r_{s}>4.2$. Table I shows that this is so for the Lang-Kohn ${ }^{5}$ exact solution of the KohnSham equations, but not for the Seidl-Brack ${ }^{3}$ restricted variational solution, which exhibits no oscillation but decreases monotonically from $\bar{n}$ inside the system to 0 outside. For the Lang-Kohn profiles, the nonmonotonicity of $n(x)$ is achieved by Friedel oscillations. For the solution of other Euler equations, it may be achieved by a single-density peak $^{20}$ inside the jellium edge.

Table II shows results for $c$ from the Seidl-Brack variational calculation, ${ }^{3}$ constructed in various ways. Equation (17) yields results that are very close to those obtained directly from an $R \rightarrow \infty$ extrapolation of the calculated ionization energy $I(R)$, while Eq. (9) yields a result very close to $-h(-\infty)$ extracted from an $R \rightarrow \infty$ extrapolation of the calculated electrostatic potential $\phi_{R}(-R)$ at the center of the cluster. Table II strongly indicates that $c \approx-0.08$ for jellium in the density range $2 \leqslant r_{s} \leqslant 6$ bohr, as confirmed by solution of the Euler equation in Ref. 2. While Eq. (17) is accurate, Eq. (9) is not.

We also tried to extract $c$ from the solution of the KohnSham equations, ${ }^{15}$ which are the Euler equations for the energy functional combining the exact $T_{s}[n]$ with the localdensity approximation for $E_{\mathrm{xc}}[n]$. We considered $r_{s}=3.93$ jellium spheres with $1<N<500$. We plotted $c(N)$ versus $N$, where $c(N)$ is given by Eq. (17) with $f(x) \rightarrow R\left[n_{R}(x)\right.$ $-n(x)]$, and found strong shell-structure oscillations (Fig. 1) around a trend line that seems to tend to -0.08 as 


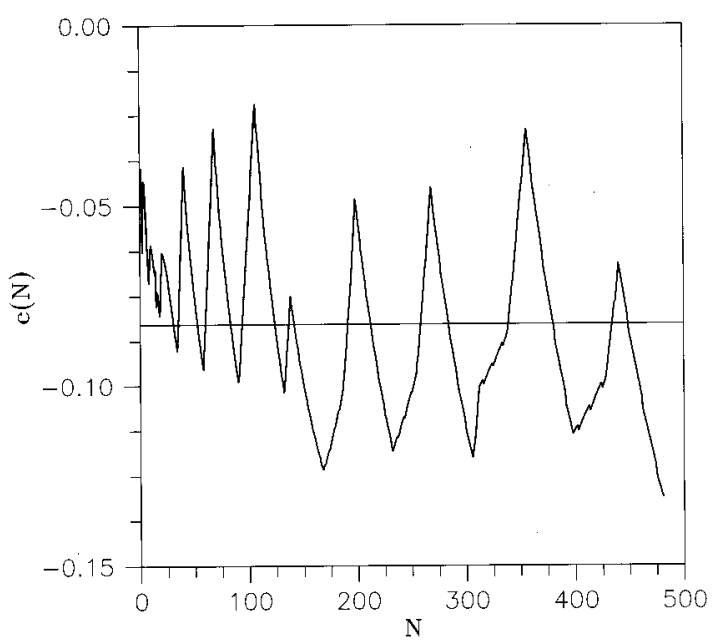

FIG. 1. A plot of $c(N)$ vs $N$ for $r_{s}=3.93$ jellium. $c(N)$ is defined by Eq. (17), evaluated with the approximation $f(x)$ $\approx R\left[n_{R}^{\mathrm{KS}}(x)-n(x)\right]$ using the Kohn-Sham profile $n_{R}^{\mathrm{KS}}(x)$ of the finite cluster with radius $R=r_{s} N^{1 / 3}$. The limiting or average value of $c(N)$ as $N \rightarrow \infty$ should be the size-effect coefficient $c$ of Eq. (1). The horizontal line at $c=-0.083$ represents the corresponding value for $c$ of Table II for $r_{s}=4$.

$R \rightarrow \infty$, with minima whenever an $n=1$ shell is filled, and maxima whenever a high- $n$ shell is filled. Plots of $R[I(R)$ $-W]-\frac{1}{2}$ oscillate even more strongly about $c=-0.1$, with maxima at the closed shells. These troublesome oscillations are of course absent from the restricted variational solution of Seidl and Brack, ${ }^{3}$ which employs the fourth-order gradient expansion for $T_{s}[n]$.

The jellium model is most realistic for $r_{s}=4$, the density at which the energy per electron minimizes. A more generally realistic model is "stabilized jellium," 21,22 which adds two terms to the energy functional of Eq. (2):

$$
\begin{aligned}
\Delta E_{R}^{\mathrm{SJ}}[n]= & \left(\epsilon_{M}+\bar{w}_{R}\right) \int d^{3} r n_{+R}(\mathbf{r})+\langle\delta v\rangle_{W S} \\
& \times \int d^{3} r \Theta(R-r)\left[n(\mathbf{r})-n_{+R}(\mathbf{r})\right],
\end{aligned}
$$

where $\langle\delta v\rangle_{W S}=-\bar{n} d e_{k \mathrm{xc}}(\bar{n}) / d \bar{n}$ vanishes at $r_{s}=4.2 \mathrm{bohr}$. The first term in Eq. (21) is purely a bulk term, and has no effect on the surface properties of a rigid cluster. Within this model, the "Koopmans" expressions become

$$
\begin{gathered}
W=\Delta \phi-\mu_{k \mathrm{xc}}(\vec{n})-\langle\delta v\rangle_{W S}=\Delta \phi-e_{k \mathrm{xc}}(\bar{n}) \\
c=-h(-\infty) \quad(\mathrm{SJ}),
\end{gathered}
$$

while the "displaced-profile change-in-self-consistent-field" expressions become

$$
\begin{aligned}
W=\Delta & \phi^{>}-e_{k \mathrm{xc}}(\bar{n})+\langle\delta v\rangle_{W S} \frac{n(0)-\bar{n}}{\bar{n}} \quad(\mathrm{SJ}), \\
c= & -\frac{2}{3} a_{s} r_{s}+4 \pi \int_{0}^{\infty} d x\left[x^{2} n(x)+x f(x)\right] \\
& +\langle\delta v\rangle_{W S} \frac{f(0)}{\bar{n}} \quad(\mathrm{SJ}) .
\end{aligned}
$$

Equation (22) is trivial from the "Koopmans" viewpoint. Equation (24) was derived in Ref. 21, and Eq. (23) in Refs. 11 and 12, while Eq. (25) belongs to the present article. The last term in Eq. (25) arises from the explicit $R \Sigma$ contribution to the last term of Eq. (21). The final term of Eq. (21) also contributes $\langle\delta v\rangle_{W S} \int_{-\infty}^{0} d x[n(x)-\vec{n}]$ to the surface energy $\sigma,^{21}$ and hence to $a_{s}$.

For solutions of the Euler equation, we may combine Eqs. (22) -(25) to find generalizations of the Budd-Vannimenus theorem. $^{13,8}$ Equating Eqs. (22) and (24) yields the stabilized-jellium-model version of the original theorem,

$$
\Delta \phi^{<}=[\bar{n}-n(0)] \frac{d}{d \bar{n}} e_{k \mathrm{xc}}(\bar{n}) \quad(\mathrm{SJ}) .
$$

Combining Eqs. (23) and (25) results in

$$
\begin{aligned}
a_{s}= & -\frac{6 \pi}{r_{s}} \int_{-\infty}^{0} d x\left\{x^{2}[n(x)-\bar{n} \Theta(-x)]+x f(x)\right\} \\
& -\frac{3}{2 r_{s}} f(0) \frac{d}{d \bar{n}} e_{k \mathrm{xc}}(\bar{n}) \quad(\mathrm{SJ}) .
\end{aligned}
$$

Within the stabilized jellium model, it is also possible to account for the self-compression of metal clusters due to surface tension, ${ }^{12,23,24}$ although we have here assumed for simplicity that the positive background is rigid.

This work was supported in part by the U.S. National Science Foundation under Grant No. DMR 95-21353, and by the Deutsche Forschungsgemeinschaft.
${ }^{1}$ J. P. Perdew, in Condensed Matter Theories, edited by J. Keller (Plenum, New York, 1989), Vol. IV.

${ }^{2}$ E. Engel and J. P. Perdew, Phys. Rev. B 43, 1331 (1991).

${ }^{3}$ M. Seidl and M. Brack, Ann. Phys. 245, 275 (1996). See also Eq. (6.10) of A. Mañanes, M. Membrado, A. F. Pacheco, J. Sañudo, and L. J. Balbas, Int. J. Quantum Chem. 52, 767 (1994).

${ }^{4}$ M. Seidl and J. P. Perdew, Phys. Rev. B 50, 5744 (1994).

${ }^{5}$ N. D. Lang and W. Kohn, Phys. Rev. B 3, 1215 (1971).

${ }^{6}$ W. Ekardt, Phys. Rev. Lett. 52, 1925 (1984); Phys. Rev. B 29, 1558 (1984).
${ }^{7}$ M. Brack, Rev. Mod. Phys. 65, 677 (1993).

${ }^{8}$ G. D. Mahan and W. L. Schaich, Phys. Rev. B 10, 2647 (1974).

${ }^{9}$ R. Monnier, J. P. Perdew, D. C. Langreth, and J. W. Wilkins, Phys. Rev. B 18, 656 (1978).

${ }^{10}$ J. P. Perdew and V. Sahni, Solid State Commun. 30, 87 (1979); J. P. Perdew, Phys. Rev. B 21, 869 (1980).

${ }^{11}$ V. V. Pogosov, Solid State Commun. 75, 469 (1990); I. T. Iakubov and V. V. Pogosov, Physica A 214, 287 (1994).

${ }^{12}$ A. Kiejna and V. V. Pogosov, J. Phys. Condens. Matter 8, 4245 (1996). 
${ }^{13}$ H. F. Budd and J. Vannimenus, Phys. Rev. Lett. 31, 1218 (1973); J. Vannimenus and H. F. Budd, Solid State Commun. 15, 1739 (1974).

${ }^{14}$ P. Hohenberg and W. Kohn, Phys. Rev. 136, B864 (1964).

${ }^{15}$ W. Kohn and L. J. Sham, Phys. Rev. 140, A1133 (1965).

${ }^{16}$ J. P. Perdew, Phys. Rev. B 37, 6175 (1988).

${ }^{17}$ J. P. Perdew, Y. Wang, and E. Engel, Phys. Rev. Lett. 66, 508 (1991).

${ }^{18}$ C. Fiolhais and J. P. Perdew, Phys. Rev. B 45, 6207 (1992).

${ }^{19}$ P. Ziesche, J. P. Perdew, and C. Fiolhais, Phys. Rev. B 49, 7916 (1994); 50, 5020(E) (1994).
${ }^{20} \mathrm{C}$. Warner, in Thermionic Conversion Specialists Conference, San Diego, 1971 (Institute of Electrical and Electronics Engineers, New York, 1972); P. Tarazona and E. Chacón, Phys. Rev. B 39, 10366 (1989).

${ }^{21}$ J. P. Perdew, H. Q. Tran, and E. D. Smith, Phys. Rev. B 42, 11627 (1990).

${ }^{22}$ A. Kiejna, Phys. Rev. B 47, 7361 (1993).

${ }^{23}$ J. P. Perdew, M. Brajczewska, and C. Fiolhais, Solid State Commun. 88, 795 (1993).

${ }^{24}$ M. Brajczewska, A. Vieira, C. Fiolhais, and J. P. Perdew, Prog. Surf. Sci. (to be published). 